\title{
A Study of Human Eyes' Detection by Window-Pair Chasing
}

\author{
Non-member \\ Member
}

\author{
Heywood Absaloms \\ Takehiko Tomikawa
}

\author{
(Kanagawa Institute of Technology, Japan) \\ (Kanagawa Institute of Technology, Japan)
}

\begin{abstract}
This paper describes a study of the detection of human eyes without employing complicated procedures. Template matching is widely utilized in supervised algorithms, however, most of these methods employ a single searching window in a limited scope. The authors take advantage of symmetry with respect to the center of the facial image and propose a pair of windows as a template. This is useful to the symmetric structure of the image data, and helpful in handling a pair of objects at once. Three window-pair templates (circular, elliptic, and square) and a single elliptic window for comparison are considered in this study. Up to six parameters (in the case of elliptic and square window-pairs) are employed to adjust the window-pair

Genetic Algorithm, based on the mechanics of probabilistic evolution, is employed to meet the multi-point search requirements without resorting to complex procedures. The algorithm, based on an unsupervised searching approach, is applied in evaluating the results from the differentiation of the image data. The differential image is used since the contours of the eye have a higher score in differentiation compared with other parts of the facial image. Some experimental simulations are made to confirm the proposed system's performance. The system is then adapted to investigate the window models listed above. The suitability of the different models in eye-detection was investigated by application to a set of images under identical Genetic Algorithm parameter settings. The detection capability of this method proved to be fairly reasonable without employing any deterministic approach, and the provided parameters in this system are easily handled regardless of the shapes of the faces in a sense.
\end{abstract}

Keywords : Feature Extraction, Genetic Algorithms, Eye Detection, Window-pair

\section{Introduction}

In human interfaces, the detection of specific parts in the facial images has attracted much interest in recent times. In order to abstract parts of a face it is very important to understand pattern recognition and facial expressions. The eyes are important in the determination of the rotation and/or orientation of the face because of their geometrical characteristics and are, therefore, considered one of the specific objects to detect in facial images.

There are two major approaches reported in the 1970s, one method of abstraction uses typical templates of parts of the face; nose, eye, mouth, and so on[1], and another method abstracts some parts of the face by their balance in a spring-connected model[2]. The former seems to be a natural way of accessing individually located parts of the face, but operates within a limited scope by using prepared dictionary patterns. The latter gives us one of the optimization procedures using dynamic programming (DP) matching technique, but requires heavy computations. While the abstraction of parts of the face has attracted a lot of interest in recent times[3]-[7], conventional eye abstraction methods are rather calculus-based or deterministic in approach and have a tendency to involve some cumbersome parameters resulting in less flexibility in system configurations.

With the background of the aforementioned shortcomings, the authors have attempted to develop a method of eye abstraction by window-pair chasing using Genetic Algorithm[8]-[10]. A deformable template to abstract facial features from images was reported[11]. This might be useful in adapting to a variety of target images by adjusting the template itself, 
but requires input images that have the features clearly in evidence. Extracting a symmetrical region from an image by Genetic Algorithm was also recently introduced[12] in which a symmetrically partitioned rectangular window is used to compare two regions and determine if a symmetrical pattern exists within these regions. This attempted to reduce the computation cost incurred in using calculus based algorithms.

This paper describes how the merits of a window-pair can be applied to eye detection in a simple way and compares the performance of different templates (elliptic pair, circular pair, square pair and single elliptic windows) in this detection. We propose a method of abstraction flexible to the facial rotation, due to the fact that the position of the eye is determined using an unsupervised chasing window-pair whose parameters are regularly adjusted. Up to six parameters (in the case of the square and the elliptic templates) are employed to adjust the window-pair, namely: the distance between the two windows, the coordinates of the template center point, the angle of the axis of symmetry, the angle of the window itself (except in circular case), and the eccentricity of the ellipses' axes, square size or circle radius. Incidentally, it contradicts our assertion of "a simple procedure", while these parameters are required to be adjusted independently and appropriately. A modified Genetic Algorithm is, therefore, employed to meet the multipoint search requirements without resorting to complex procedures.

\section{Basic Concepts}

\subsection{Image Pre-processing}

The contours of the eye have a higher score in differentiation compared with other parts of the facial image. This is a useful key in distinguishing the eye area from others without having any prior knowledge about eyes. We also found that in most cases the reflecting surface of the eye had the greatest slope and pixels having a high gradient concentrated on the eye region of the face as shown in Fig. 1. For facial pictures, edge abstraction done using a differential calculus operation has a tendency to concentrate points on parts of the face of great slope. In this tendency, the eyes especially are regions where big slopes concentrate.

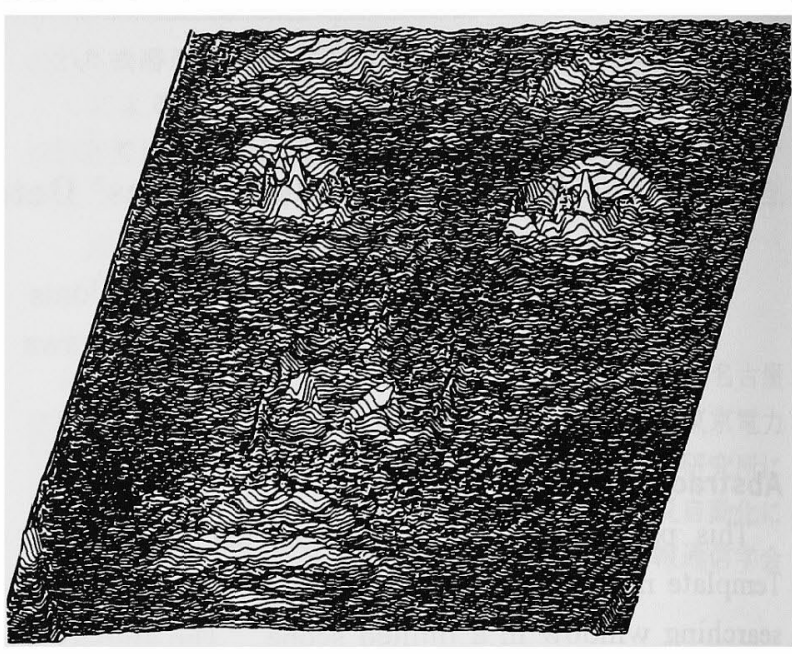

Fig. 1. Example of Sobel's Differentiation

In order to further eliminate regions or features of the facial image that are not necessary to our method for abstraction, a cut-off threshold had to be determined In this investigation several facial images were used in determining a reasonable value for this threshold which, though not necessarily universal to all possible images, would work well within our range of images. The procedure to obtain the threshold started with the application of Sobel's first order differential operation in horizontal and vertical directions to the images The resulting histograms were then examined and the resulting differential images analyzed. The facial picture has 256 levels of gray image and by changing the cut-off threshold, we examined what facial parts are clearly recognizable at different threshold levels From the set of images the eye, nose, and mouth are found to concentrate within the top $2 \%$ of the population in the slope density histogram, with an average at $1.4 \%$. From these results, the study establishes the slope density characteristics limiting threshold $S$ by the upper $1.4 \%$ of differential image slope values for every image.

In this study, using window templates to locate the eyes, this slope density information is used as an evaluation measure in the abstraction of the eyes with very promising results. 


\subsection{Models}

In recent times Genetic Algorithms (GAs) are being used to solve large dimension optimum value search problems. The search procedure using the GA technique is simple and has the advantage that it is possible to obtain results in a realistic calculation time and so in this research we use different models and a modified GA search in 4 to 6 dimensions in the abstraction of the eye. The descriptions of the models follows.

\subsubsection{Window-pair Model}

We define a window-pair $W_{1}$ and $W_{2}$ for use in searching for the two eyes, and the facial picture $l$. The ellipse-window-pair, for example, has a structure as shown in Fig. 2 and is used to search for the pair of eyes in this case approximated by ellipses

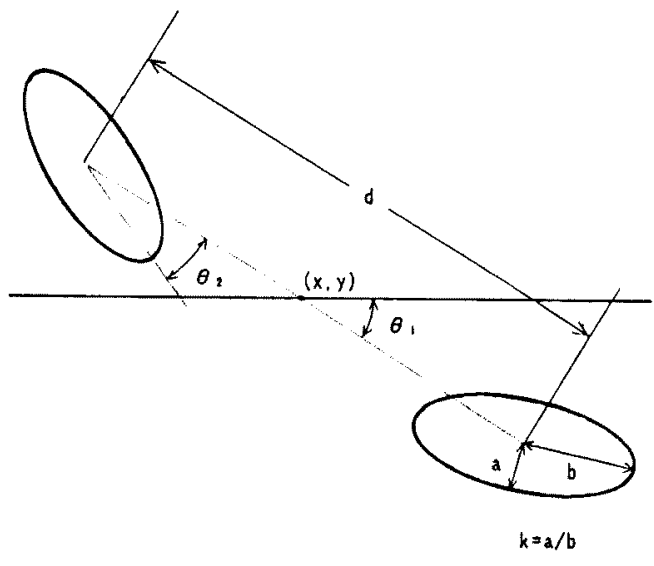

liig. 2. Window-pair Model

To decide the posture and position of this search model, we search in a total of six dimensions, namely, the coordinates $(x, y)$ of the midpoint of the segment joining the centers of the two identical elliptic windows $W_{1}$ and $W_{2}$, the model's rotation angle $\theta_{1}$, the centerto-center window separation distance $d$, the ellipses' eccentricity $k$, and the approximation of the ellipses' slant angle $\theta_{2}$.

The circular window pair search is in 5 dimensions the coordinates $(x, y)$ of the midpoint of the segment joining the centers of the two identical circular windows $W_{1}$ and $W_{2}$, the model's rotation angle $\theta_{1}$, the center-to-center window separation distance $d$, and the circle radius $r$. The window slant angle $\theta_{2}$ has no application in this case.

The square widow pair search is in $\mathbf{6}$ dimensions : the coordinates $(x, y)$ of the midpoint of the seginent joining the centers of the two identical square windows $W$, and $W_{2}$, the model's rotation angle $\theta_{1}$, the center-to-center window separation distance $d$, the square size $w^{\prime}$ and the approximation of the squares' slant angle $\theta_{2}$.

Due to the assumed symmetry of the face, the window slant angle $\theta_{2}$ is identical to both $W$, and $W$.

\subsubsection{Single Window Model}

The elliptic window was selected for the single window model. The single elliptic window model used to abstract the eyes is identical to any one of the windows shown in Fig. 2 with the dimensions as described below. The search is in $\mathbf{4}$ dimensions: the coordinates $(x, y)$ of the centroid of the ellipse, the model's rotation angle $\theta_{1}$, and the ellipse's eccentricity $k$. The single window search proceeds in two stages, first one eye is located and then the Genetic Algorithm is reset and the search repeated to locate the second eye but with the already located area removed from the search area. The search for both eyes was done so as to give a better comparison of performance against the window-pair models

\subsection{Models' Adaptation to Generic Algorithm}

We define the genes in the GA as the elements of a bit sequence. For the ellipse pair we have a 6 dimension bit sequence $\left(x, y, \theta_{1}, d, k, \theta_{2}\right)$; for the circular pair a 5 dimension sequence $(x, y, \theta, d, r)$; for the square pair the 6 dimension sequence $\left(x, y, \theta_{1}, d, n_{1}, \theta_{2}\right)$ and for the single ellipse window the 4 dimension sequence $\left(x, y, \theta_{1}, k\right)$. A large number of individuals having these genes is generated, and the generation process repcated obeying the $\mathrm{G} \wedge$ hereditary rules in order to abstract the eyes. Various factors determine the number of bits allocated to each of the parameters (genes) within the chromosome. Among these factors are the size of the search range desired for the parameter and the accuracy to which the parameter is to be determined. In this study the search was entirely in the integer domain and so the gene size was determined primarily by the search range. 


\subsection{Evaluation Function}

\subsection{Window-pairs}

The edge accumulation degree is used as the search's evaluation factor; and the evaluation function $f_{1}$ is defined as

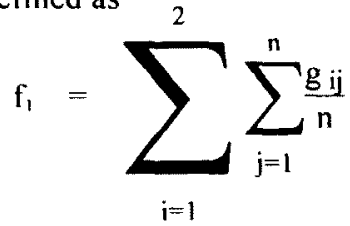

and if $\left(g_{i i}<S\right)$ then $g_{i i}=0$;

where $g_{t \prime}$ is the value of differentiation at the location in window $i, n$ is the number of pixels in the window, and $S$ is the slope density characteristics' limiting threshold. In the search, the windows $W_{1}$ and $W_{2}$, must obey the condition $W_{1} \in I ;\{i=1,2\}$

We therefore define the evaluation function $\boldsymbol{f}_{2}$ by the formula

$$
\mathrm{f}_{2}=\left\{\begin{array}{l}
0:\left(W_{1} \in I\right) \\
P:\left(W_{i} \notin I\right)
\end{array} ;\{\mathrm{i}=1,2\} \ldots\right.
$$

where $P$ is a negative number denoting penalty for boundary violation and chromosomes receiving penalty die by taking on this negative value. We must also consider the penalty for symmetry violation between the two windows as

$$
\mathrm{f}_{\mathbf{1}}-\left\{\begin{array}{l}
0:\left|\sum_{\mathbf{j}=1}^{\mathrm{n}} \mathrm{g}_{1 \mathrm{j}}-\sum_{\mathrm{j}=1}^{\mathrm{n}} \mathrm{g}_{2 \mathrm{j}}\right| \leq R \\
P:\left|\sum_{\mathbf{j}=1}^{\mathrm{n}} \mathrm{g}_{1 \mathrm{j}}-\sum_{\mathbf{j}=1}^{\mathrm{n}} \mathrm{g}_{2 \mathrm{j}}\right|>R
\end{array}\right.
$$

where $R$ is the symmetry constant. The evaluation function of the chromosome's adaptation to its environment $\mathbf{f}$, is then expressed as the sum of the Eqns. (1) to (3) i.e

$$
f=f_{1}+f_{2}+f_{3}
$$

The eye abstraction problem thus becomes that of maximizing the evaluation function $\mathbf{f}$ in Eqn.(4) During the search the fitness of an individual is determined according to the genes in the evaluated chromosome. Such genetic material is modified and improved through the evolution of three basic operations of $\mathrm{G} \wedge$; reproduction, crossover and mutation

\subsubsection{Single Window}

The edge accumulation degree is again used as the search's evaluation factor; and the evaluation function $\dot{f}_{1}$ is defined as

$$
f_{1}-\sum_{j-1}^{n} \frac{g j}{n}
$$

and $\quad$ if $\left(g_{i}<S\right)$ then $g_{i}=0$;

where $g$, is the value of differentiation at the location, in the window, $n$ is the number of pixels in the window, and $S$ is the slope density characteristics' limiting threshold.

In the search, the window $W$ must obey the condition $W \in I$. We therefore define the evaluation function $f_{2}$ by the formula

$$
\dot{r_{2}}-\left\{\begin{array}{l}
0:(W \in I) \\
P:(W \notin I)
\end{array}\right.
$$

where $P$ is a negative number denoting penally for boundary violation and chromosomes receiving penalty die by taking on this negative value. Since there is only one window there is no penalty for symmetry violation and the evaluation function of the chromosome's adaptation to its environment $f$, is then expressed as the sum of the liqns.(5) and (6) i.c

$$
\dot{f}=f_{1}+f_{2}
$$

The eye abstraction problem thus becomes that of maximizing the evaluation function $f^{\prime}$ in Eqn.(7). During the search the fitness of an individual is determined according to the genes in the evaluated chromosome. Such genetic material is modified and improved through the evolution of three basic operations of $\mathrm{GA}$; reproduction, crossover and mutation.

\subsubsection{Fitness Function Sensitivity to Area}

The behavior of the fitness function with respect to the area of the model window has a critical bearing to the performance of the system as a whole. This is 


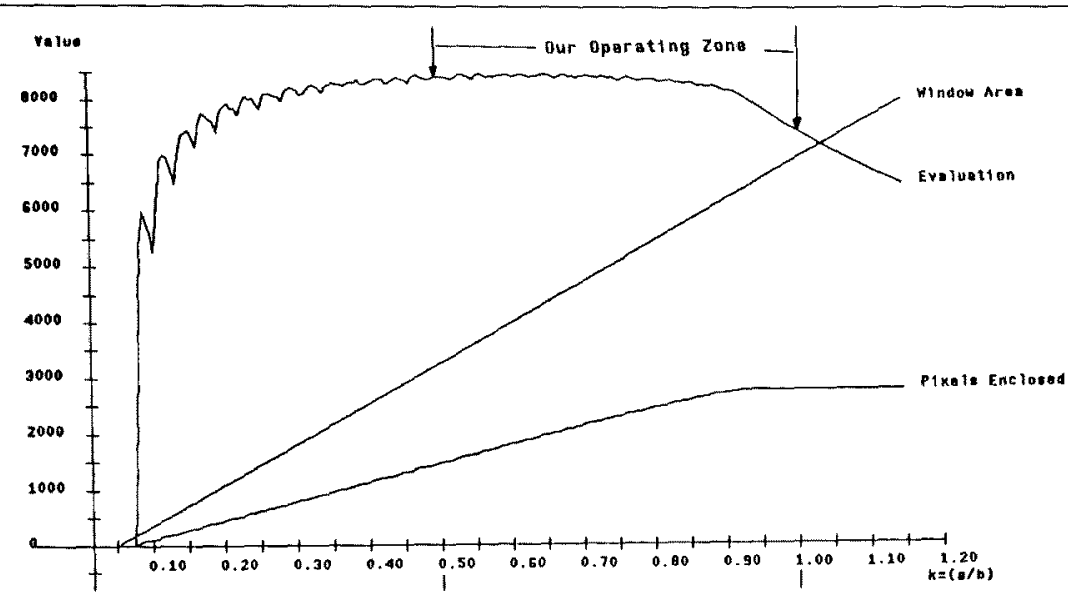

Fig. 3. Ellipse Window Evaluation Curves

because progression through the search, generation by generation, is based on the performance of the various chromosomes (individuals) and the performance is quantified solely by the fitness function. The different window shapes behave differently in evaluation as the area varies even for the same location on the image and offers some insight into the evolution of the different windows during a search as well as possible reasons for their tendency toward some solution as the optimum solution. By varying the size of the window placed concentric with a circular region of maximum differential score to model the eye area in an actual search, this variation was investigated. In the real search the search windows are expected to gravitate to the center of gravity of the eyes and thus the testwindow positioning in this investigation. The dimensions of the windows are varied to cover the regions used in the actual search. The results for the Ellipse, Circular, and Square windows when the circular region has a radius of 30 pixels are shown in Fig. 3, 4, and 5 respectively. Kach figure contains three curves for ; the variation of the window area, the variation in the number of scoring pixels contained within the window and finally the variation of the evaluation score for the window. On the vertical axis is the score and for the horizontal axis the variable that determines the area of the window.

For the ellipse window (Fig. 3) the horizontal axis represents the ellipse eccentricity $k$, and varies up to 1.10; the width of the ellipse is kept comstant at os pixels. The evaluation score curve characteristics exhibit three major sections; region I from the origin to about $k^{-0}=30$, region 2 comprising the final smooth section from about $k=0.90$, and region 3 that lies in between regions 1 and 2 . In region 1 the area of the window and the number of pixels are of the same magnitude and small changes in size lead to sometimes big swings in evaluation score. There are no scoring pixels enclosed until after approximately $k^{-0} 0.08$ after which the curve rises sharply from 0 (zero). The enclosed scoring pixels do not increase in direct proportion to the area and fairly large fluctuations are seen on the curve. This region has no practical application as the window is too small (flat) to give meaningful results. Within region 2 the number of scoring pixels remain the same as the window area increases due to the fact that the window entirely encloses the region of differential score. In this region GA optimization can take place with the window size being fitted more tightly around the high scoring location in the image as it climbs up the slope from the right. Region 3 characteristics lie in between regions 1 and 2. The curve is not entirely smooth but also not as rough as in region 1. A search in this region, especially the latter portion, is practical as the window size is adequate and there is a slight slope that the optimization algorithm can climb. A search that starts from the left has also some chance of progressing up the slight slope from left to right in this region

For the circle window (Fig. 4) the horizontal axis represents the circle radius $r$, and varies from $/ 10+5$ pixels. The evaluation curve characteristic can again be divided into three regions. Region 1 from $r=1$ to about $r=15$ pixels, region 2 consisting of the smooth 
section at the end from $r=30$ pixels and region 3 that lies in between the regions 1 and 2 . The characterization of the regions and their applicability in a search are similar to those for the corresponding regions for the ellipse window (Fig. 3)

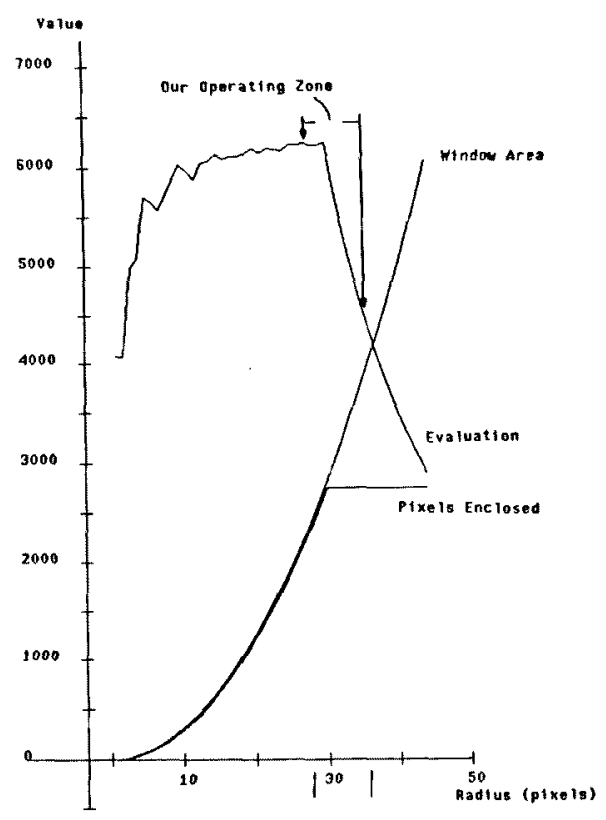

Fig. 4. Circle Window Evaluation Curves

For the square window (Fig. 5) the horizontal axis represents the square width $w$, and varies from 2 (1) 90 pixels. Once again the evaluation curve characteristic can be divided into three regions. Region 1, the flat section from $w=2$ to about $w=43$ pixels, where the number of enclosed pixels equals the area of the window; region 2 comprising the end section from $w=60$ to $w=90$ pixels, where the number of enclosed scoring pixels remains constant as the window size increases; and finally region 3 that lies in between the two regions 1 and 2 . Region 1 of the curve for the square is not of much use in the search as the evaluation remains constant while window size changes. Apart from the constant evaluation score the region in fact olfers a reason why square window searches that start from the left are likely to fail as the region has the highest score while not necessarily being the optimum. This is especially true when a very tiny window (small $w$ ) closes in on a similarly tiny section of the image having a few high scoring pixels, that could be anywhere in the image. This region must be avoided.
Regions 2 and 3 offer good search zones especially for searches starting from the right.

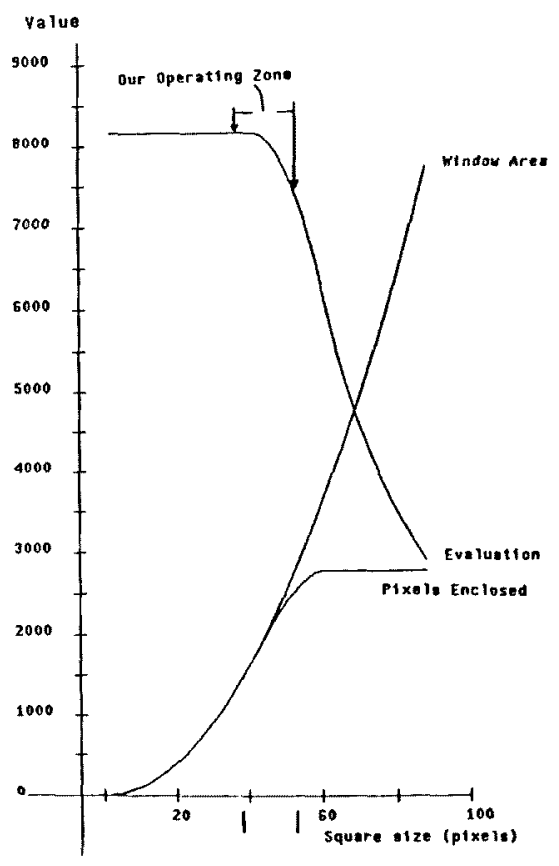

Fig. 5. Square Window Evaluation Curves

The regions of the evaluation curves that would correspond to our search area are indicated on the diagrams. These assume window location at the center of gravity of the eye region which is also assumed to be homogeneous. The three curves above indicate that the ellipse and circle windows would be best for searches that can come from either side (left or right) while the square window search would be more suited for searches that always begin from the right. In actual searches it was noted that the circle window searches especially, normally started from the right; the ellipse window search to a slightly lesser degree and the square window search least consistent in this regard.

\subsection{4}

Sample of Actual Distribution

The procedure in 2.4 .3 above was then applied to a typical differential image with the test-window being centered at the center of gravity of the area immediate to the eye. The center of gravity was determined from an image of the eye region after differentiation and thresholding (using S). Fig. 6 shows the position of the center of gravity for each eye. 


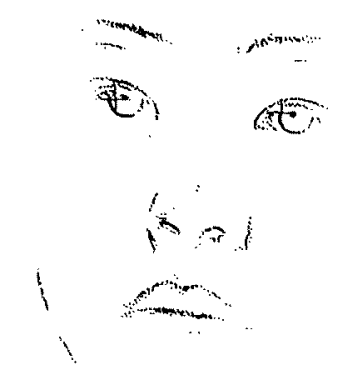

Fig. 6. Centre of Gravity of Eyes

From the diagram it may be noted that the center of gravity lies in the neighborhood of the highest differential scores and not at the center of the eye ellipsoid as may have been expected

The results of the investigation on both eye regions are shown in Figs. 7 to 12 for the Circular, Square and Ellipse windows respectively. Based on the actual size ranges of our windows, our operation region is once again indicated on the curves. The operating region does not include the maxima on the curves which represent windows too tiny to be of practical use in the search considering the size of the eyes (similar to regions 1 and part of region 3 in 2.4 .3 above).

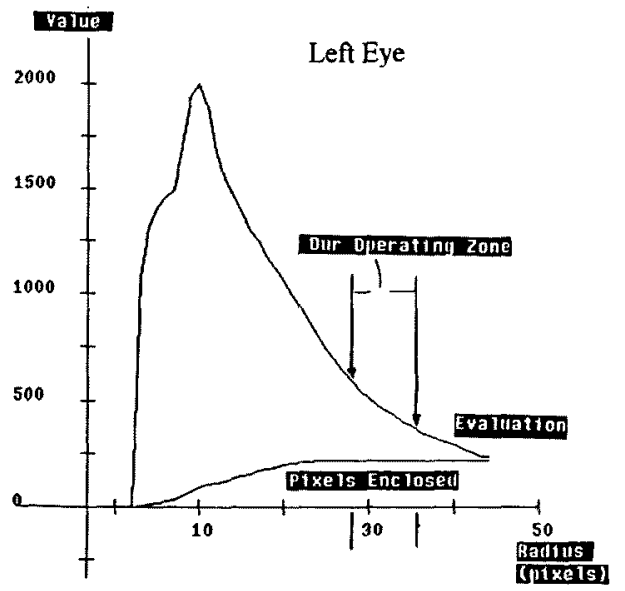

Fig. 7. Circle Window Evaluation Curve for Left Eye

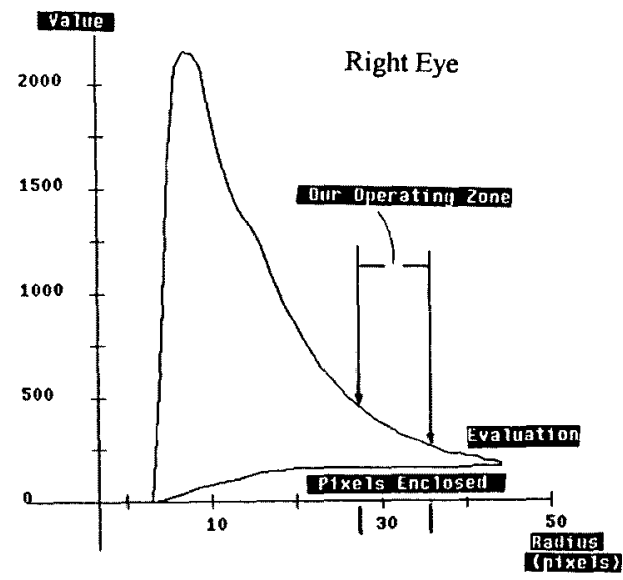

Fig. 8. Circle Window Evaluation Curve for Right Eye

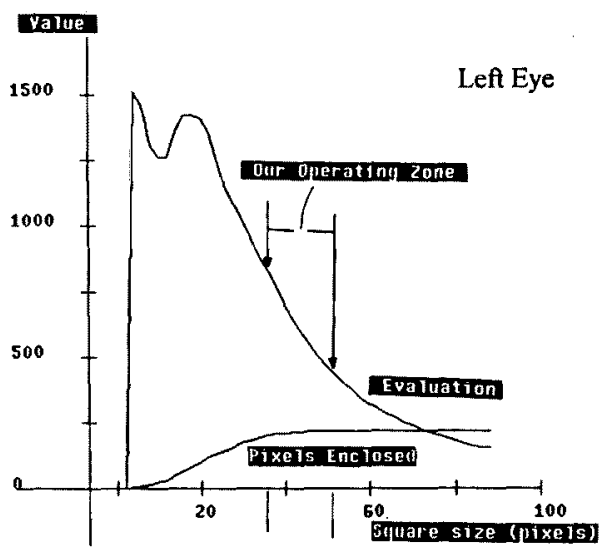

Fig. 9. Square Window Evaluation Curve for Left Eye

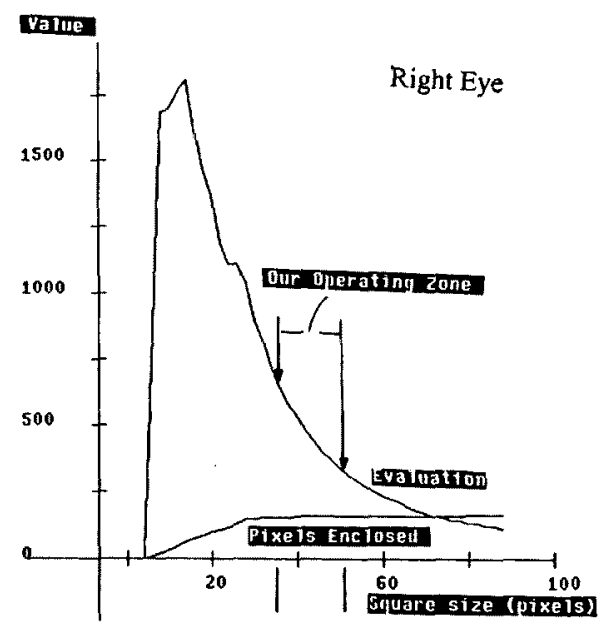

Fig. 10. Square Window Evaluation Curve for Right Eye 


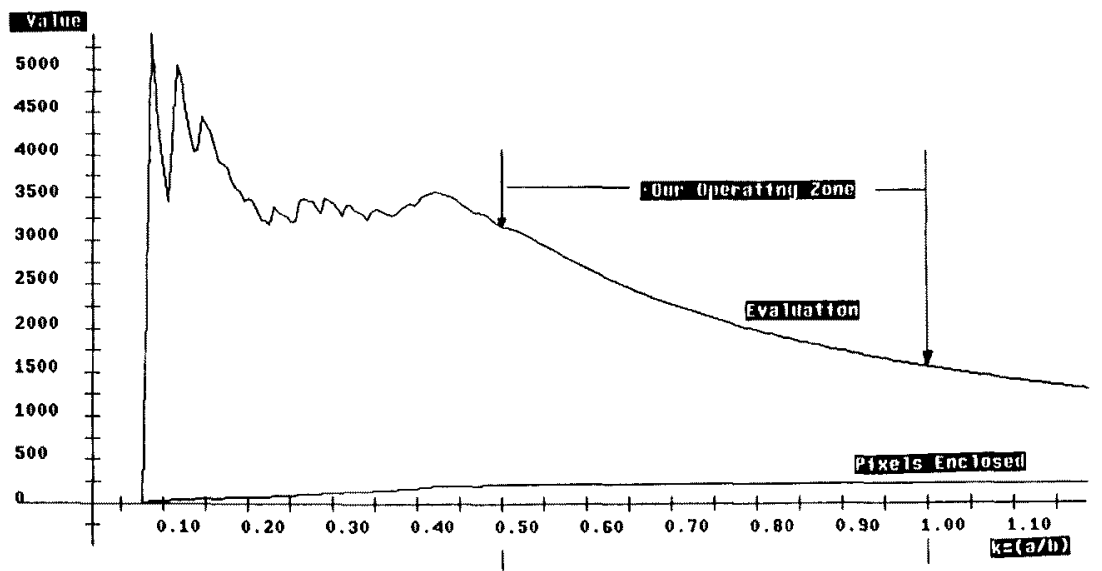

Fig 11. Ellipse Window Livaluation Curve for left Eye

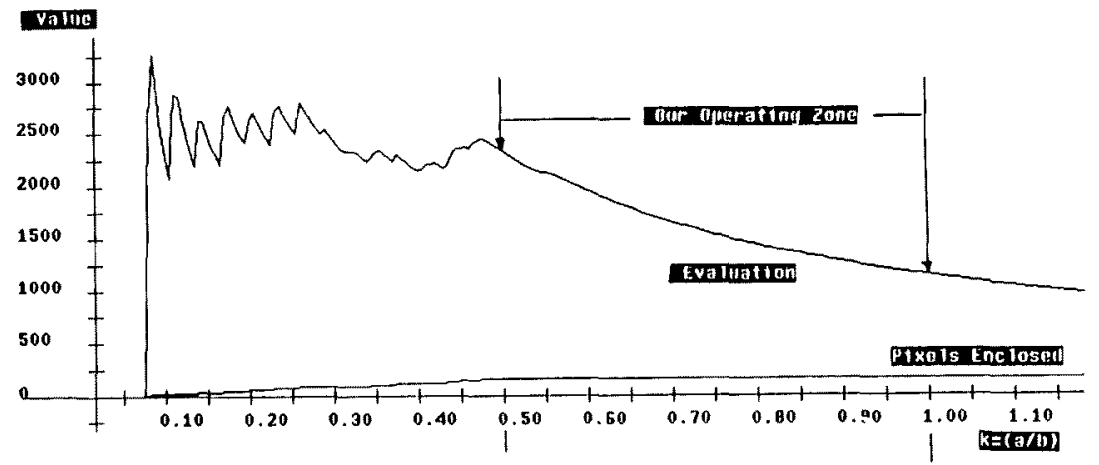

Fig. 12. Ellipse Window Evaluation Curve for Right Eye

\section{System Configuration}

\subsection{Structure of the Chromosomes}

Each chromosome has a certain length and is a vector of the form $\left[c_{1}, c_{2}, c_{3 . .}\right]$ where each $c_{1}$ is a gene consisting of binary digits $\{0,1\}$. When the genes are implemented in contiguous bits then the chromosome takes the structure shown in Fig. 13 for the case of the ellipse window pair (the actual implementation in this study was different as is explained in 3.3 .1 below.)

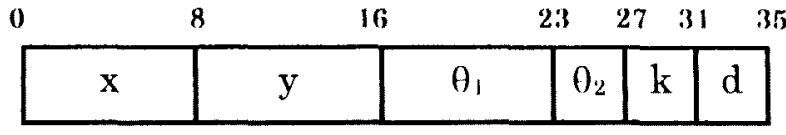

Fig. 13. Chromosome Structure

The contents of the chromosome fully identify the size, position and posture of the search model. The evaluation of individual chromosomes in a population is defined as the normalized value of the edge accumulation score of the pixels within the inodel window(s) plus the penalty for violating image boundary conditions or image symmetry conditions (Eqns.(4) and (7)). The fitness value of the chromosome must reflect both the quality of similarity and quantity of fit of the model window(s) it defines.

\subsection{System Flow Chart}

Considering the system flow chart (Fig. 14), the image pre-processing stage for the system consists of Sobel fillering and threshold level determination. After the image pre-processing the parameters for the GA are entered. The main system procedure then begins with the random generation of the initial chromosome population and the random determination of the search starting point. The rest of the process consists of a closed loop process which is the model window chasing for an optimum solution. 


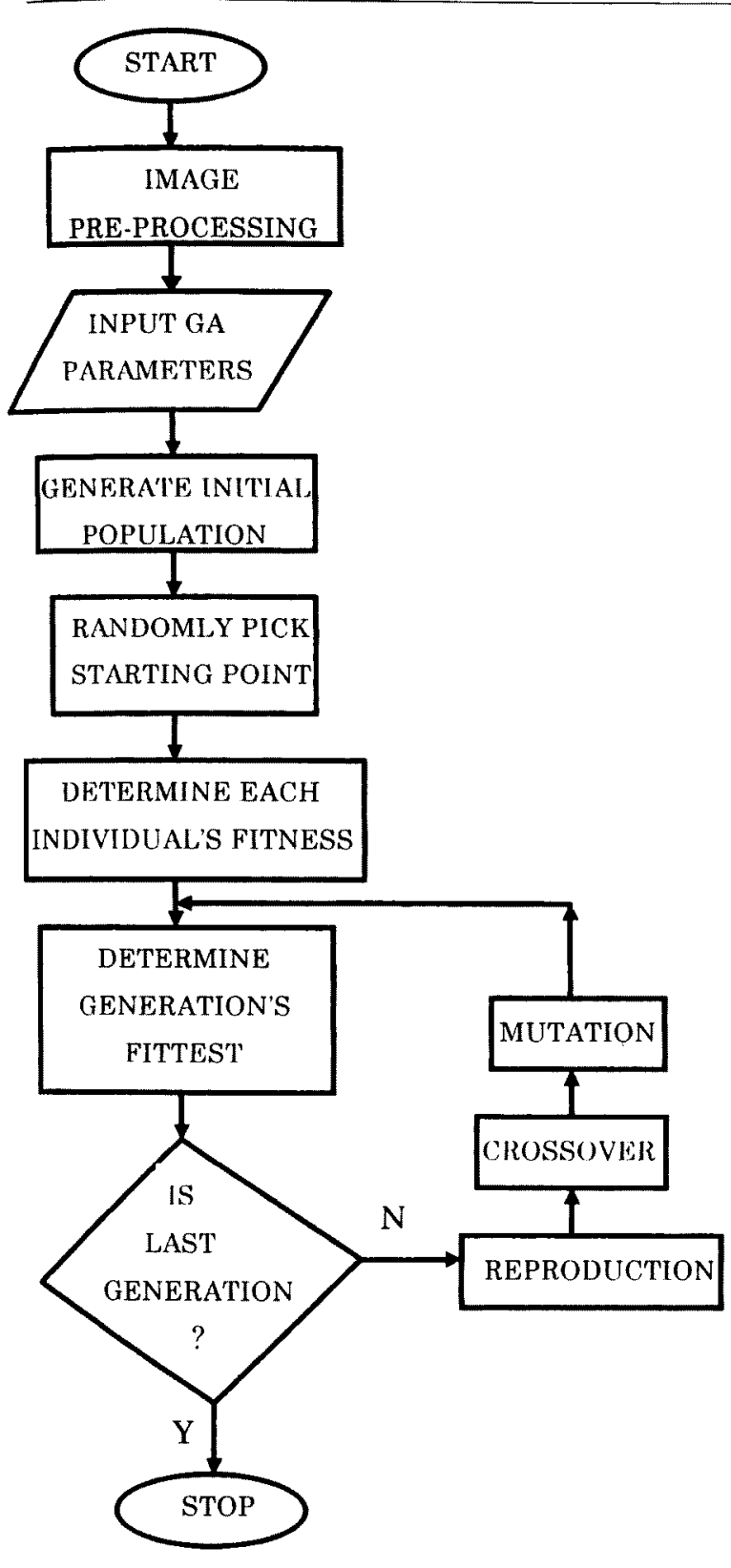

Fig. 14. Flow Chart

Subject to the evaluation function as a measure of goodness detection that we want to maximize, lluree basic (i^ operations; reproduction, crossover and mutation, must be activated. A stopping critcrion halts the process after a fixed number of evolutions of the population and takes the best solution found.
This system operation may be classified into two phases as

(1) The model is created according to the conditions assumed for a typical human face,

(2) The model is moved around the input image according to the rules of GA and within the constraints of Eqns (4) and (7).

\subsection{The Modified GA}

The alleles (bits composing the genes) were randomly scattered about the chromosome. This was in order to overcome the effects of a possibly biased random number generator in deciding the crossover points. Having the alleles representing any one of the variables scattered through the chromosome ensured the GA crossover operation affected all the variables. An example distribution is shown in Fig. 15. The search was found to proceed more efficiently with the scattered alleles than with the case of the contiguous alleles.

The standard GA progression through generations was modified to achieve a more rigorous search. A strategy was implemented to keep in play the best performing chromosome that had been seen through all prior generations of the search. While enabling a more rigorous search of the solution space, through the use of high mutation rates, this strategy ensures that the best performing chromosome propagates through the generations and is never lost. The procedure is as follows. A record is kept of the contents of the best performing chromosome and this chromosome is reintroduced into the population whenever it has a higher fitness value than the top chromosome for that generation. The re-introduction is done by the replacement of the poorest performer for the generation by the best chromosome just before reproduction operation is carricd out. If the top chromosome for the generation has a higher fitness value than the best chromosome then the top chromosome becomes the best chromosome and no changes are made to the population. This strategy generally led to higher final

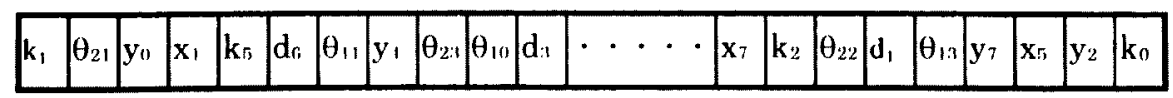

Fig. 15. Scattered Alleles Chromosome Structure 
fitness values (implying more efficient searcles!) and about 15\% higher success rate over the standard implementation.

Apart from the above strategy a second (annealing) was introduced in an attempt to escape local maxima during the search. Whenever there was stagnation in the search over a number of successive generations (say 4) a given percentage (say 90\%) off the bottom end of the population was subjected to a very high rate of mutation (say uniform mutation of 70\%) before proceeding to the next generation. This increases the chance of introducing new and better genes to the population. Note that since the ' $\wedge$ dd Best' strategy is in place and the top (say 10\%) performers in the population are untouched the advances made thus far are never lost and the result of the search can only improve.

\section{Experiments}

Experiments were carried out to confirm the validity of this method of eye detection. The original image we used was a $(256 \times 256)$ pixel facial image given a level transformation. The population used in the algorithm was 130 and we searched through 100 generations. This time we used simple GA and the detailed heredity rule was omitted. In investigating the validity of the method the elliptic window-pair was used. Preliminary experiments were carried out to determine the optimum values for the Genetic Algorithm parameters after which the performance of the method over many trials was done.

In the 6 dimension search if we use 3 parameter control of position and posture under the constraint $W$, $\in \quad I ;\{i=1,2\}$, the search range is decided naturally The parameters $\left(d, k, \theta_{2}\right)$ of the search range depend on the face geometrical characteristics and these differ from person to person but can be restricted considering actual human faces. The search range was therefore decided from examination of several facial images. We used the search ranges below.

- The spacing between the eyes $d$, with an average value of 120 and $\pm 20 \%$ as the search range, i.e. $d$ $=120 \pm 24$ [pixels]

- The ellipses' eccentricity $k$, a 4-bit gene of the range equal to $0.5+(4$-bit $) / 32$ i.e. $k=0.5 \sim 1.0$
- The square size $w$, a 4-bit gene of the range equal to $45 \pm(4-b i t)$ pixcls i.c. $w=38 \sim 52$ pixcls.

- The circle radius $r$, a 4-bit gene of the range equal to $32 \pm(4-$ bit $)$ pixels. i.e. $r=28 \sim 36$ pixels

- The slant of the eye is decided on a 4-bit gene and the search range we decided on was $\theta_{2}=+7^{\circ} \sim$ $8^{\circ}$ represented in 2 's complement notation.

NB. The size variations for the different shapes was chosen so that on the average the largest dimension for all the models would be approximately equal.

In comparing of the performance of the four inodels a total of 27 different images were used. For each image each model was started off with the same starting population of chromosomes. At least ten (10) searches per model per image were done, each time with a different starting population. The results over all these searches was then compiled to give an indication of the relative performances.

\section{Experimental Results}

\subsection{Sample Results}

From the system validity check, sample results are shown in Figs. 16(a) $\sim(\mathrm{g})$. Figs. 16(a) and (b) show the original image, Figs. $16(\mathrm{c})$ and (d) the image after differentiation and Figs. 16(e) and (f) the results of the abstraction. The overall results gave a success rate of over $98 \%$ on the trial images. By employing images of faces rotated in the horizontal and vertical axis it is noted that reasonable abstraction results are still obtained under these conditions as long as a good portion of the each of the eyes is visible.

l'o show the validity of the evaluation function threshold parameter $S$, the result obtained when $S$ is not used is shown in Fig. $16(\mathrm{~g})$. By introducing $S$ the unnecessary slopes are removed and we oblain a better result. Similar results are also seen when GA does not properly converge within the given number of generations.

In comparing the four models some of the positive results are shown in Fig. $17(\mathrm{a}) \sim(\mathrm{c})$ (for images $8 i, 80 \%$, and $7 i$ respectively) and some failures in Fig. 18(a) $\sim(c)$ (for images 2 and $3 o r$ respectively.) 


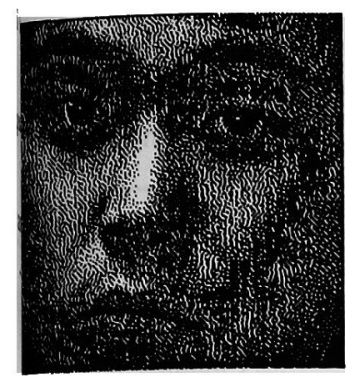

(a)

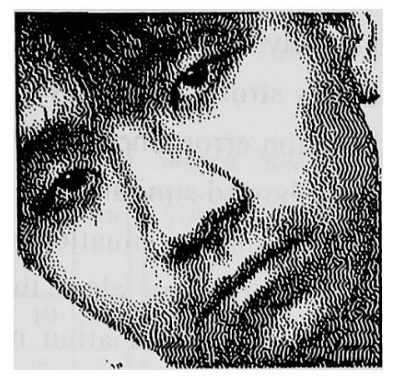

b)

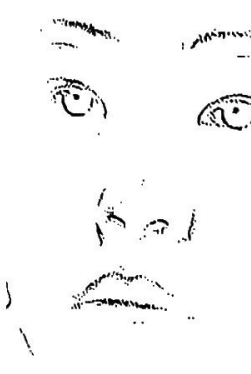

(c)

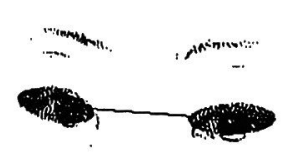

$$
1
$$

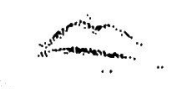

(e)

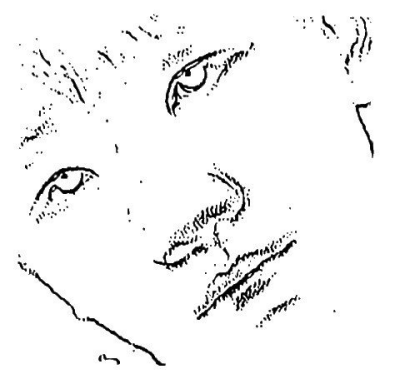

(d)

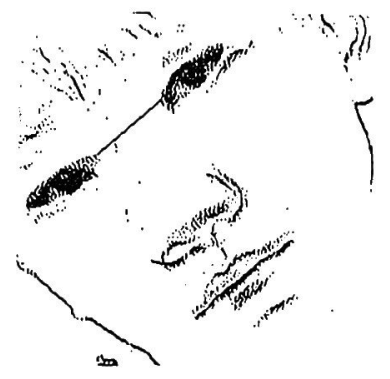

(f)
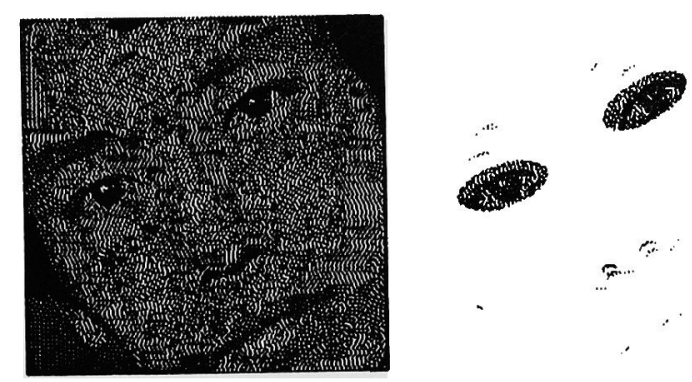

(a)
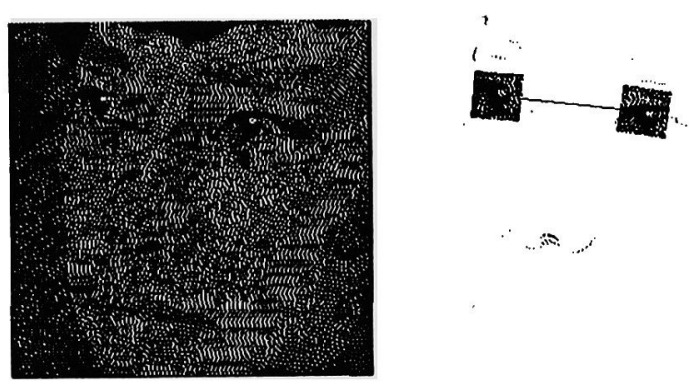

(b)
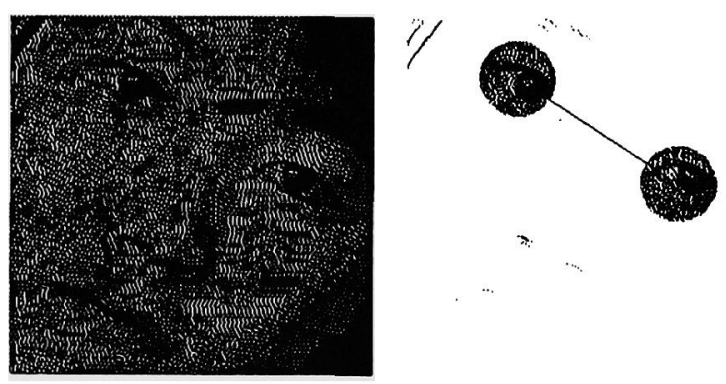

(c)
Fig. 17. Sample Window Comparison Results - Success

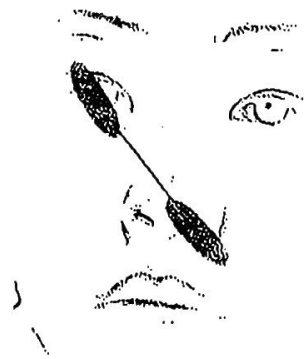

(g)
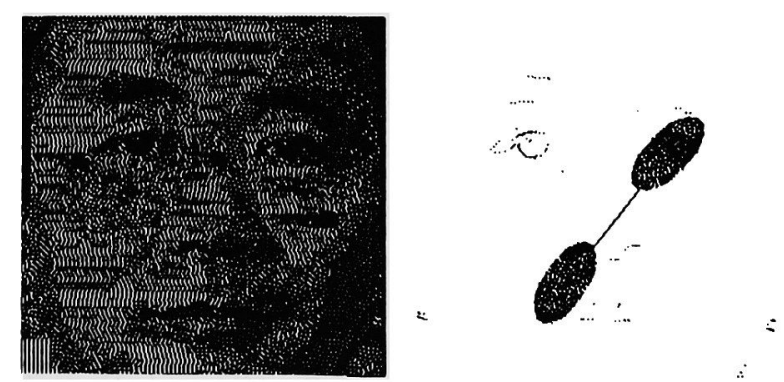

Fig. 16. Sample Experimental Results

Fig. 18(a). Sample Window Comparison Results - Failures 


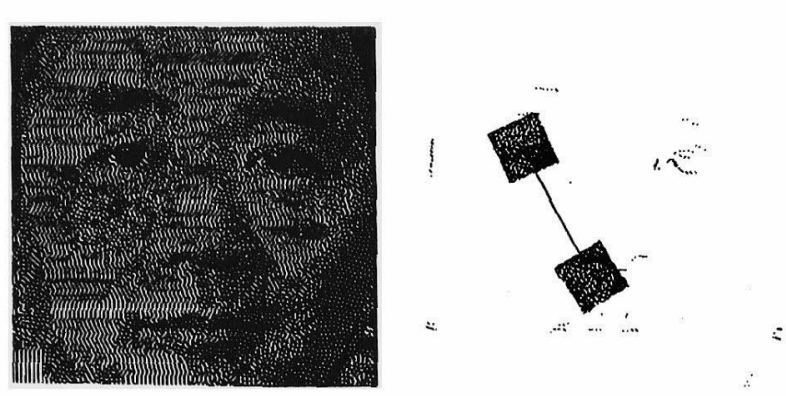

(b)
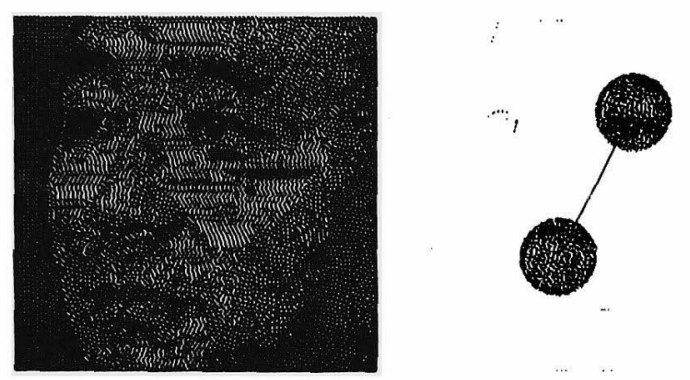

(c)

Fig. 18. Sample Window Comparison Results - Failures

Consolidated results from the comparison of the four models are shown in Tables 1 . below. Only entries of the number of failures are made, a blank slot indicates no failure for that image.

The results for the images 2 and $3 o r$ deserve comment. In image 3 or the image preprocessing resulted in only faint outlines of the eyes remaining and a comparably strong outline of one of the nostrils. This led to detection error since the two eye regions and the nose region showed similar score on evaluation.

In image 2 a similar situation arose but in this case though the regions are clear they are once again of similar strength on evaluation making it easy for the system to fault to the nose region as one of the eyes. So for images in which the spatial distances between the two eyes and the nose are similar and the differential image has a comparably strong result in the nose region as the eye regions, a higher than average percentage of failures would be expected.

Certain image types present a problem to the system, e.g. $2 i$ and tor in Fig. 19(a) and (b) respectively.

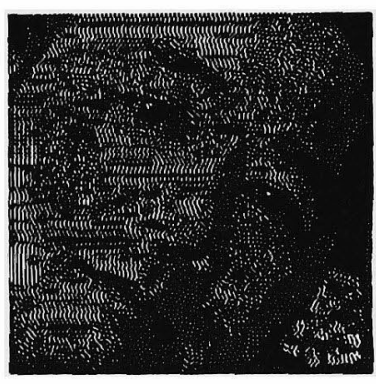

(a)

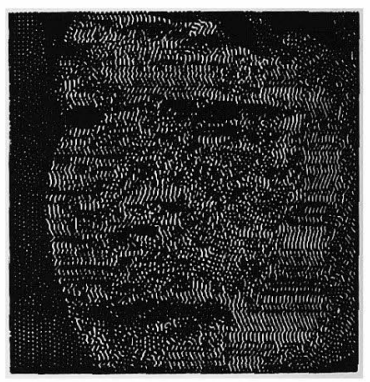

(b)
Fig. 19. Problem Images

Table 1. Comparison : Failures out of 10 Searches

\begin{tabular}{|c|c|c|c|c|c|c|c|c|c|c|c|c|c|c|c|c|c|c|c|c|c|c|c|c|}
\hline & \multicolumn{24}{|c|}{ Ima ge } \\
\hline Model & 2 & 3 & $.3 i$ & $3 o r$ & 4 & $4 i$ & 5 & $5 j$ & $50 r$ & 6 & $6 i$ & 6 in & 7 & $7 i$ & $70 r$ & 8 & $8 i$ & $8 o r$ & 9 & $9 i$ & $9 o r$ & 10) & \begin{tabular}{|l|l|}
101 & $100 \mathrm{r}$ \\
\end{tabular} & 11 \\
\hline Circle Window Pair & 3 & & & 2 & 1 & & & & & & & & & & & & & & & & & & & \\
\hline Ellipse Window Pair & 1 & & & 3 & & & & & & & & 1 & & & & & & & & & & & & 1 \\
\hline Square Window Pair & 2 & & & 5 & 4 & & 3 & & & & & & & & 3 & & & & & & & 1 & & 2 \\
\hline Eye-by-Eye Single Ellipse & 1 & & & 2 & & & & & & & & & & & & & & & & & & & & \\
\hline
\end{tabular}

\begin{tabular}{|c|c|c|c|}
\hline & Success & Rate & Summary \\
\hline Model & Total 7rials & No. Fail & \% Success \\
\hline Circle Window Pair & 250 & 6 & $97.6 \%$ \\
\hline Ellipse Window Pair & 250 & 6 & $97.6 \%$ \\
\hline Square Window Pair & 250 & 20 & $92.0 \%$ \\
\hline Eye-by-Eye Single Ellipse & 250 & 3 & $98.8 \%$ \\
\hline
\end{tabular}


Table 2. Comparison : Problem Images

\begin{tabular}{|c|c|c|c|c|c|}
\hline \multirow[b]{2}{*}{ Model } & \multicolumn{2}{|c|}{ Image } & \multirow{2}{*}{$\begin{array}{l}\text { Success } \\
\text { Total } \\
\text { Thials }\end{array}$} & \multirow{2}{*}{$\begin{array}{l}\text { Rate } \\
\text { No. } \\
\text { Fail }\end{array}$} & \multirow{2}{*}{$\begin{array}{c}\text { Summan } \\
\% \\
\text { Succes }\end{array}$} \\
\hline & $2 i$ & $40 r$ & & & \\
\hline Cincle Window Pair & 10 & 9 & 20 & 19 & $6 \%$ \\
\hline Ellipse Window Pair & 8 & 7 & 20 & 15 & $25 \%$ \\
\hline Square Window Pair & 8 & 10 & 20 & 18 & $10 \%$ \\
\hline Eve-by-Eye Single Ellipse & 10 & 10 & 20 & 20 & $0 \%$ \\
\hline
\end{tabular}

The search results from these images for the four models are seen in Table 2 and sample images in Fig. 20(a) $\sim(d)$.

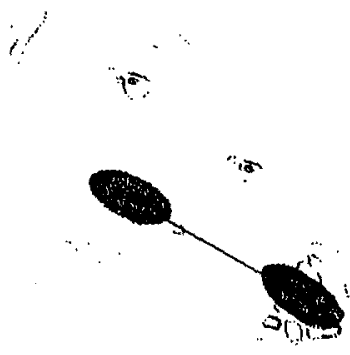

(a)

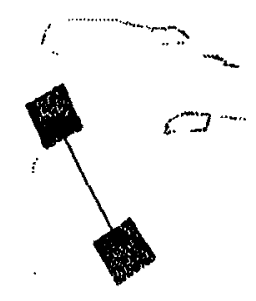

(c)

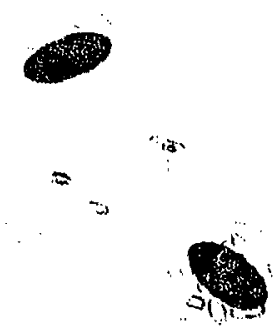

(b)

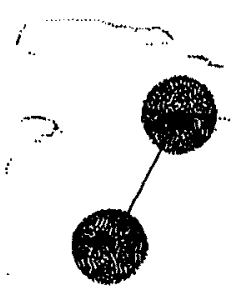

(d)

Fig. 20. Problem Images' Results

The reasons for the poor performance for the two inages differ and are representative of a class of images under which the system would not perform optimally. Image $2 i$ illustrates the effect of a significantly noisy background that leads to high scores under differentiation. This sort of background has the effect of drawing the search from the eyes and thus failure. The image 4 or on the other hand has little of the eyes, especially the right eye, showing and result in the differential score for the other parts of the face being higher than that for the eyes. In this particular case the search fails by taking the nose area as one of the eyes. The versatility of the elliptic window in comparison with the others is however still evident even in this kind of images. The possible reason for which is the close conformance to the general shape of the eyes (and their differential image) and the ability to follow the orientation of the eyes on the face unlike the other shapes. These two factors makes for a better maximization of the differential score within the window area.

The single-window search method now shows its major weakness, that of lacking the further constraint of adhering to syınınetry in the search. Thus even in the case where there is no possibility of placing the second cye given the position of the first eye, the single window search is no wiser. The window-pair searches however would discriminate against such positions and the chromosomes pertaining to the position would die out. So while the window-pair may disregard a stronger showing area in locating the second eye and pick the weaker area by symmetry constraints and thus succeed, the single window model searching without the symmetry constraint fails. The importance of the symmetry constraint (Eqn.3) in this abstraction is thus illustrated.

\subsection{Conclusion}

We proposed an unsupervised abstraction of symmetrical objects by a 6 dimension search utilizing window-pair chasing. Then a Genetic Algorillm ( $(\mathrm{i} \wedge)$ is used to mect our multi-point searching solution of the six parameter problem. Will the proposed system a pair of eyes was abstracted regardless of the rolation of the image. The system's present chromosome configuration handles vertical axis image rotation of up to $\pm 63^{\circ}$ but simply by allocating one more bit for $\theta$, the full range of $\pm 90^{\circ}$ can be achieved.

The detection capabilities of the method are fairly reasonable without resorting to complicated procedures. Difficulties in detection arose when the differential score for the eye regions were comparable to those of the nostril region and the spatial distances between the two eyes and the eye to nose distances are comparable. 
lmages with clattered backgrounds or where the percentage of the eye visible is small also present abstraction difficulties. In the study, the image preprocessing (Sobel filtering and thresholding) greatly reduces the effect of the eyebrows in the final image and any residual traces are so insignificant, compared to the eye region, as to present no problem during the eye abstraction.

Further work in improving the usefulness of the proposed system include 1) resolving the window-tonose error problem, 2) improving the alignment of the search window's major axis with the major axis of the eye, 3 ) improving the alignment of the centroid of the search window and the centroid of the eye, and 4) implementing a more flexible method for determining the image limiting threshold.

The study of the different window models in the search gives indications of the difference in performance of the different models under similar conditions. Possible reasons for difference in performance are offered by considering the evaluation score variations for the different window-shapes, located at the image center of gravity, while adjusting their sizes. Single window detection while showing slightly better performance than the best window-pairs falls short in the quality of the detection since the windows are independent and their final posture was often uncorrelated with the posture of the eyes unlike the window-pairs.

(Manuscript received December 20, 1995,

Revised May 13, 1996)

\section{References}

11] T. Sakai, et al, "Processing of Multi-leveled Pictures by Computer - The Case of Photographs of Human Face -," Truns. IECL, 54-C, 6, 1971. , pp445-452.

[2] M. A. Fischler, R. A. Elschlager, "The Representation and Matching of Pictorial Structures," ILELE Truns. C-22, I, 1973., pp67-92.

[3] S. Sakamoto, et al, "Extracting Feature Points on Human Eye Photographs," Proc. MIRUI'92, July 1992., ppII-143-150.

[4] Y. Sumi, Y. Ohta, "Human Face Analysis Based on Distributed 2D Appearance Models," Trans. IFICE, J77-D-II, 12, Dec. 1994., pp2342-2352.

[5] M. Hoguro, et al, "The Detection Method for the
Characteristic Points of II uman Face based on Artificial Neural Networks and its Evaluation," Proc. IEICE, PRU94-75, Dec. 1994., pp23-30.

[6] L. C. Desilva, et al, "Face Orientation Estimation," Proc. of IEICL, PRU94-18, 1994., ppI7-24.

[7] R. Brunelli, T. Poggio, "Face Recognition : Features versus Templates," Ililil: Trans. PAMI15, Oct. 1993., pp1042-1052.

[8] T. Yamagish, T. Tomikawa, "An Extraction of IIuman Eyes by GA," Report of II'S, 7L,-2, Oct. 1993. , pp2-185-2-186.

[9] T. Yamagish, T. Tomikawa, "A Detection of Human Eyes in Facial Images by Pair-Window Searching," Report of IElCE, D-522, Mar. 1995. pp248.

[10]11. Absaloms, T. Tomikawa, "A Detection Of Human Eyes by Window-Pair Chasing," Proc. of Ist KSWHVl: International conference, Aug. 1995.

[11]A. L. Yuille, "Deformable Templates for Face Recognition," Journal of Cognitive Neuroscience, Vol.3, No. I., pp59-70.

[12]Shirakawa, T. Nagao, "Extraction of symmetrical regions in still images using a genetic method," Tech. Report of IEICE, PRU95-161, 199511.,pp19-24.

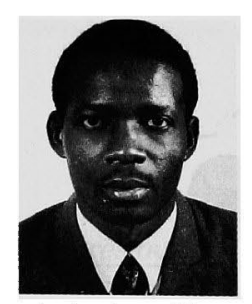

Heywood Absaloms

Born January 23, 1965. Received B.Sc. degree from University of Nairobi, Kenya in 1988 and M.E degree from the University of Technology, Sydney (UTS), Australia in 1993. Presently a Ph.D research student at Kanagawa Institute of Technology Graduate school. Current research area is Genetic Algorithm Applications in Image Processing. Member of the Institute of Electronics, Information and Communication Engineers and the IEEE.

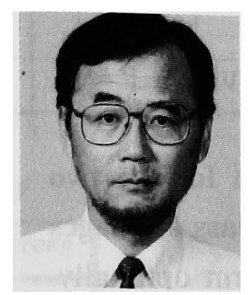

Takehiko Tomikawa

Born February 1, 1945. Received M.E and Ph.D degrees from Shizuoka University in 1979 and 1982 respectively. Presently professor of Electrical Engineering at the Kanagawa Institute of Technology Electronic Engineering Department. Doctor of Engineering. Current research interests include image processing and the application of Genetic Algorithms. Member of Information Processing Society of Japan and the IEEE. 